Trauma Berufskrankh 2006 • 8[Suppl 3]:S271-S27 6 DOI 10.1007/s10039-005-1065-z

Online publiziert: 13. Dezember 2005

๑) Springer Medizin Verlag 2005
H. Martin · D. Da Silva Ferreira - R. Markoll

Institut für innovative Medizin, München

\title{
Pulsierende Signal Therapie als Behandlungsmodalität bei Arthrose
}

\section{Wissenschaftliche Grundlagen}

In den 1970er und 1980er Jahren wurde in experimentellen Studien erstmals gezeigt, dass in Zellgeweben unter physiologischen Bedingungen elektrische Potenziale und Ströme entstehen können [2, 5, 9]. Es wurden In-vitro-Untersuchungen durchgeführt, um den Einfluss von pulsierenden elektromagnetischen Feldern auf Knorpelzellen bzw. Knorpelgewebe zu untersuchen $[2,7,11]$. Tierexperimentelle Untersuchungen beschäftigten sich vorwiegend mit der Produktion der extrazellulären Matrix (EZM) $[1,18,29]$.

Auch in Knochengewebe sind ähnliche elektrophysiologische Vorgänge bekannt. Bei Kompressionen wird die kristalline Struktur der Hxdroxylapatite im Knochengewebe deformiert, wobei ein piezoelektrischer Strom entsteht [15]. In der EZM wird somit ein mechanischer Stimulus über eine physikalische Transduktion in ein „Strömendes Potenzial“ umgewandelt. Die biologischen Vorgänge, die einerseits die permanent ablaufenden Knochenumbauvorgänge regulieren und andererseits zur Knochenheilung führen, sind bislang noch nicht vollständig aufgeklärt.

Vergleichsweise ähnliche, aber mit einer Reihe von Unterschieden behaftete Vorgänge gelten für Knorpelgewebe [34]. Nach dem heutigen Wissenstand ist dieses in der Lage, Kraftstöße zu absorbieren und eine annähernd gleichmäßige Kraftumverteilung innerhalb des knorpelüberzogenen Gelenkareals sowie auf die darunter liegenden knöchernen Strukturen [4, 19,
$27,28,30,35] \mathrm{zu}$ ermöglichen. Nur durch ein Zusammenwirken aller mechano-elektro-chemischen Eigenschaften der EZM erhält Knorpelgewebe seine besondere Fähigkeit. Bei jedem Kompressionszyklus wird die EZM einer zeitlich und räumlich variierenden Druckbelastung ausgesetzt, wobei es zu Flüssigkeitsverschiebungen und dadurch auch zu Verschiebungen der darin befindlichen Ionen kommt. Begleitet von hydrostatischen und osmotischen Druckveränderungen fließen gelöste, vorwiegend positiv geladene Ionen an den fixen negativen Ladungen der sulfatierten Proteoglykanmatrix vorbei und erzeugen sowohl innerhalb als auch außerhalb der EZM „Strömende Potenziale“ $[14,19,20$, 21, 25, 33]. Gerade wegen dieser Zusammenhänge ist die Diskussion, welche spezifische Information auf den Chondrozyten bei mechanischer Belastung übertragen wird, um dessen Metabolismus zur Aufrechterhaltung und Reparatur des Knorpel- gewebes zu stimulieren, zurzeit noch offen. Aus diesen Gründen lag es nahe, die Anwendung von pulsierenden elektromagnetischen Feldern auch auf die Behandlung von Arthrosen auszuweiten.

\section{Grundlagen der Pulsierenden Signal Therapie (PST)}

Sie ist ein nichtinvasives, bisher nebenwirkungsfreies elektrophysiologisches Behandlungsverfahren. Sie baut auf theoretischen, experimentellen und klinischen Erkenntnissen der pulsierenden elektromagnetischen Felder (PEMF) auf, die zur Behandlung von Pseudoarthrosen eingesetzt werden [17]. PST ist allerdings eine besondere Form der Therapie mit PEMF $[31,32]$ : Es wird ein Spulensystem verwendet, das bei wechselnden Feldstärken im Bereich von 0,5-1,5 mT mit Frequenzen zwischen 1 und $30 \mathrm{~Hz}$ impulsmodulierte, annährend homogene Magnetfelder er-
Abb. 1 Variables PSTGerät, stationärer ringförmiger Applikator

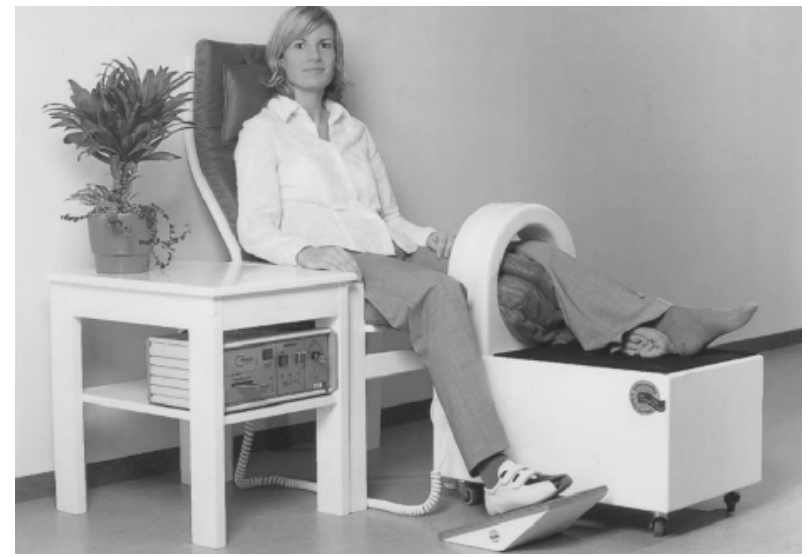




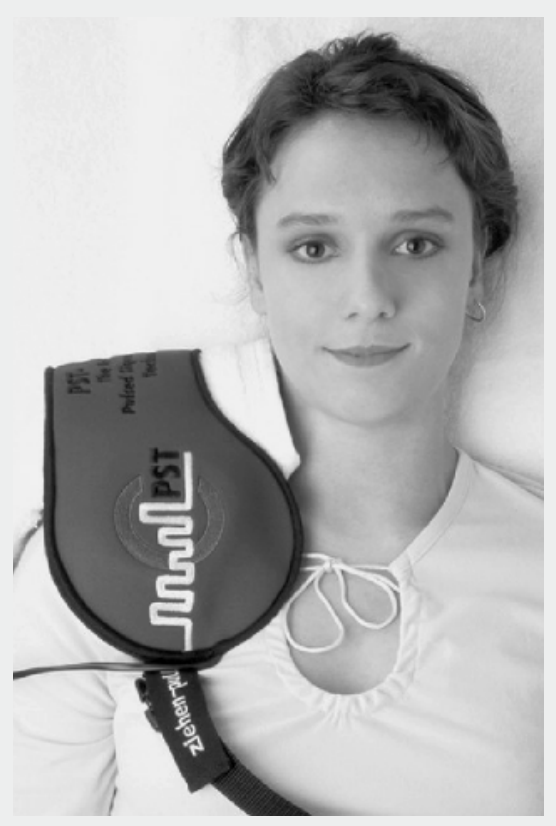

Abb. $2 \triangle$ Mobiles PST-Gerät, mobiler beweglicher Applikator

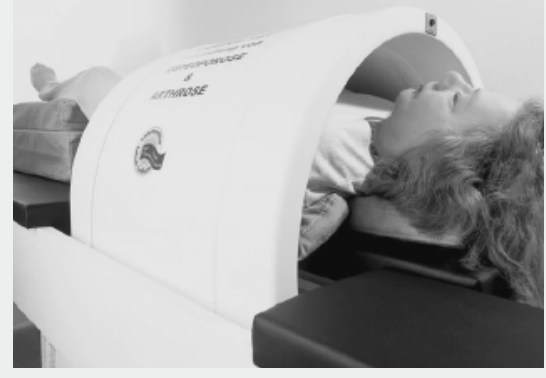

Abb. $3 \triangle$ PST-Liege
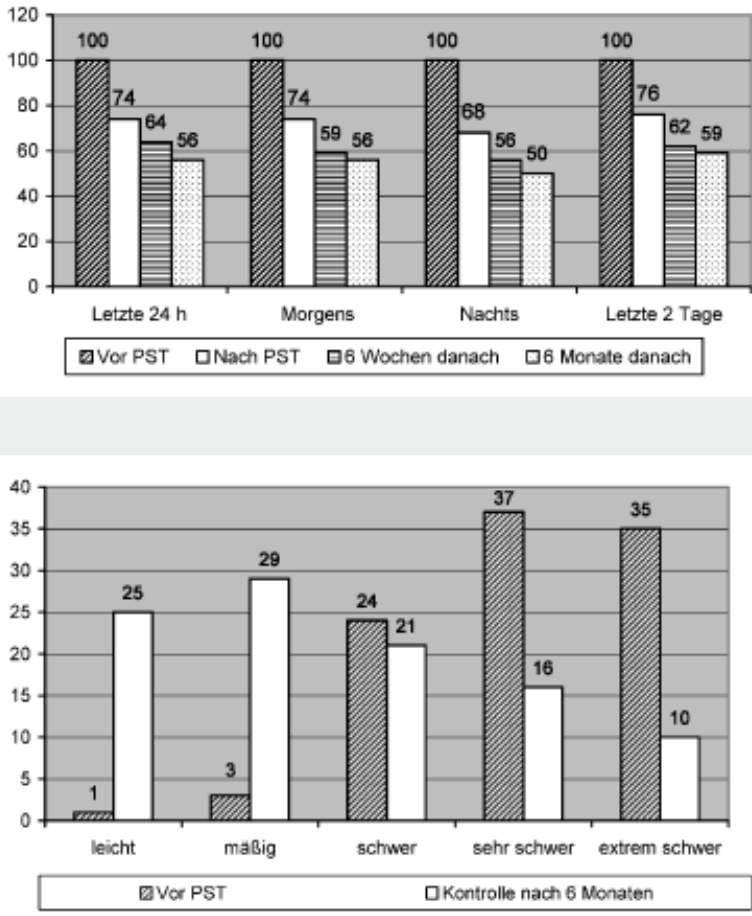

Abb. $5<$ Häufigkeitsverteilung der Gonarthrosescores [\%]

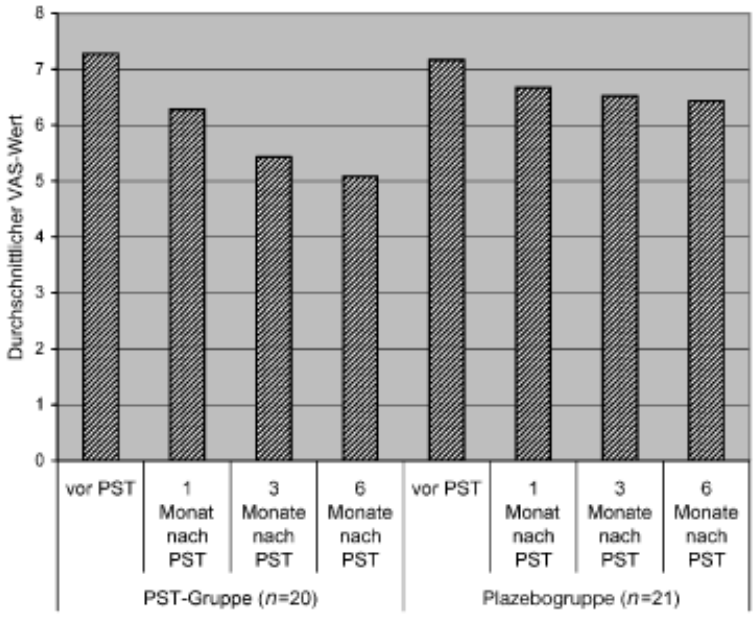

Abb. $6<$ Durchschnittlicher VAS-Wert zeugt. 25 klinische Studien und 5 In-vitroStudien haben die Wirkung der PST auf Bindegewebe untersucht $[8,12,13,16,22$, $31,32,36]$. Sie soll die eigenen physiologischen Signale imitieren und dadurch die Regeneration und Reparatur im Bindegewebe des Bewegungsapparats, einschließlich Knorpelgewebe, stimulieren und entsprechende Heilungsprozesse beschleunigen $[8,12,13,16,22,31,32,36]$.

Die Indikation zur Therapie ist vorwiegend die Arthrose - bis zum Stadium III nach Kellgren. Kontraindikationen zur Anwendung des Verfahrens sind das Vorliegen von Tumoren im Behandlungsfeld,
Gravidität oder das Tragen empfindlicher elektrischer Geräte wie Herzschrittmacher oder Kochleaimplantate.

\section{PST-Therapie von Gelenken}

Bei allen peripheren Gelenken werden 9 einstündige Behandlungen durchgeführt, wobei 2 unterschiedliche Geräte zum Einsatz kommen können, entweder ein stationärer ringförmiger (• Abb. 1) oder ein mobiler beweglicher Applikator (• Abb. 2). Die Wirbelsäule, die Hüftgelenke und der Schultergürtel werden 12 Behandlungsstunden unterzogen, die auf der Ganzkör- perliege durchgeführt werden (• Abb. 3). Als Block- oder Kurbehandlung wird diese Behandlungsserie mit in der Regel $1 \mathrm{~h} /$ Werktag, maximal 2 Therapiestunden täglich, über 1-2,5 Wochen durchgeführt. Therapiepausen sollten nur an den Wochenenden bestehen. Diese Behandlungsschemata haben sich empirisch als am wirkungsvollsten erwiesen.

\section{Klinische Studien im Bereich der Gonarthrose}

Erste prospektive, randomisierte, plazebokontrollierte Doppelblindstudien an 
der Yale-Universität, USA, zeigten 1993 bei Arthrosen der Kniegelenke (86 Patienten) und degenerativen Veränderungen der Halswirbelsäule (81 Patienten) eine hoch signifikante therapeutische Wirkung in der tatsächlich behandelten Patientengruppe gegenüber der unbehandelten Kontrollgruppe [37]. Dabei waren die Ergebnisse beider Patientengruppen in der Nachuntersuchung 1 Monat nach Therapieabschluss deutlich ausgeprägt und z. T. hoch signifikant. Dies ist wahrscheinlich darauf zurückzuführen, dass die unbehandelte Gruppe während des Therapiezeitraums wie erwartet einen gewissen Plazeboeffekt zeigte. Dieser war bereits $1 \mathrm{Mo}-$ nat nach Ende der Therapie kaum mehr nachweisbar, sodass dann signifikante Unterschiede festgestellt werden konnten, beispielsweise mit $p=0,001$ für die Angabe der Schmerzstärke bei der Gonarthroseverumgruppe zu $p=0,07$ bei der Gonarthroseplazebogruppe im Vergleich zur „baseline“.

1998 wurde im Hôpital Cochin, Paris, eine prospektive, randomisierte doppelblinde Studie mit insgesamt 40 Patienten mit Gonarthrose nach den Kriterien des ACR durchgeführt. Sie zeigte deutliche Unterschiede zwischen der Verum(21 Patienten) und der Plazebogruppe (19 Patienten). Zur Nachkontrolle 3 Monate nach Behandlungsende zeigten beide Gruppen statistisch signifikante Unterschiede bei der Schmerzstärke (VAS o10) mit $p<0,01$ und bei dem erhobenen Lequesne-Index mit $p=0,02$.

Eine deutsche prospektive multizentrische Studie zur Behandlung von Gonarthrosen (Stadium II und III nach Kellgren) mit insgesamt 303 Patienten aus 49 deutschen PST-Zentren wurde von Professor Dr. Rainer Breul, Ludwig-Maximilians-Universität, München, überwacht und 2000 in der Zeitschrift „Orthopädische Praxis“ veröffentlicht [12]. Bei der Kontrolle 6 Monate nach Beendigung der Therapie konnte bei etwa $73 \%$ der Patienten eine signifikante Befundbesserung festgestellt werden. Statistisch signifikant zeigten sich eine Reduktion der Knieschmerzen (• Abb. 4) und Verbesserungen bei den erfragten Alltagsaktivitäten wie Gehen, Treppensteigen usw. Nebenwirkungen wurden nicht beobachtet. Die Einstufung der Patienten nach dem Lequesne-

Trauma Berufskrankh 2006 • 8[Suppl 3]:S271-S276

DOI 10.1007/s10039-005-1065-z

c) Springer Medizin Verlag 2005

H. Martin · D. Da Silva Ferreira · R. Markoll

Pulsierende Signal Therapie als Behandlungsmodalität bei Arthrose

\section{Zusammenfassung}

Seit 1996 wird die pulsierende Signaltherapie als biophysikalisches Behandlungsverfahren bei degenerativ bedingten Beschwerden des Bewegungsapparats eingesetzt. Nach einem empirischen Behandlungsschema werden 9 oder 12 einstündige Behandlungen mit speziellen Geräten verabreicht. Technisch gesehen sind die pulsierenden Signale elektromagnetische Rechteckimpulse mit variierenden energetischen Parametern. Durch die Behandlung sollen verstärkt Regenerations- und Reparationsvorgänge im arthrotischen Knorpelgewebe und dem gelenkumgebenden Bindegewebe ausgelöst werden. Die vorliegenden GCP-konformen klinischen Studien und Verifikationsuntersuchungen zeigten positive Effekte hinsichtlich Schmerzsituation und Funktionsfähigkeit der behandelten Gelenke. Nach den vorliegenden Ergebnissen sind diese Resultate signifikant bes-

\section{Pulsed Signal Therapy as a treatment modality in osteoarthritis}

\section{Abstract}

Pulsed Signal Therapy (PST) is a biophysical therapy that has been used since 1996 for the treatment of degenerative joint disorders. The therapy involves 9 or 12 one-hour treatment sessions with special medical devices. The pulsed electromagnetic signals are quasirectangular in waveform and their energy parameters vary. The pulsed signals stimulate the repair and regeneration of arthritic cartilage and surrounding connective tissue. Clinical trials following Good Clinical Practice guidelines have demonstrated a statistically significant positive effect of PST in reducing pain and improving joint function for a minimum of 6-12 months compared ser als Plazebobehandlungen und halten mindestens 6-12 Monate an. Durchgeführte In-vitro-Untersuchungen stützen die Hypothese einer Steigerung von Aufbauvorgängen in den Strukturen der extrazellulären Matrix. Weitere Untersuchungen zur Abklärung einer evtl. vorliegenden chondrogenen Wirkung sind erforderlich. Nach den bisherigen Erfahrungen kann die Pulsierende Signal Therapie als nichtinvasives, bisher nebenwirkungsfreies Behandlungsverfahren eine Erweiterung des konservativen Therapiespektrums bezüglich der Behandlung degenerativ bedingter Beschwerden des Bewegungsapparats darstellen.

\section{Schlüsselwörter}

Pulsierende Signal Therapie . Elektromagnetisch · Arthrose

Chondrozyten · Knorpelgewebe

\section{to placebo. In vitro studies have supported the hypothesis that PST stimulates chondro- cyte growth and increases structural com- ponents of the extracellular matrix. Prospec- tive studies to clarify the effects of PST on chondrocytes and transcription factors are warranted. PST, as a painless non-invasive treatment with no known side effects, wi- dens the range of conservative treatment options for degenerative joint disorders.}

\section{Keywords}

Pulsed Signal Therapy · Electromagnetic . Osteoarthritis · Chondrocytes · Cartilage 


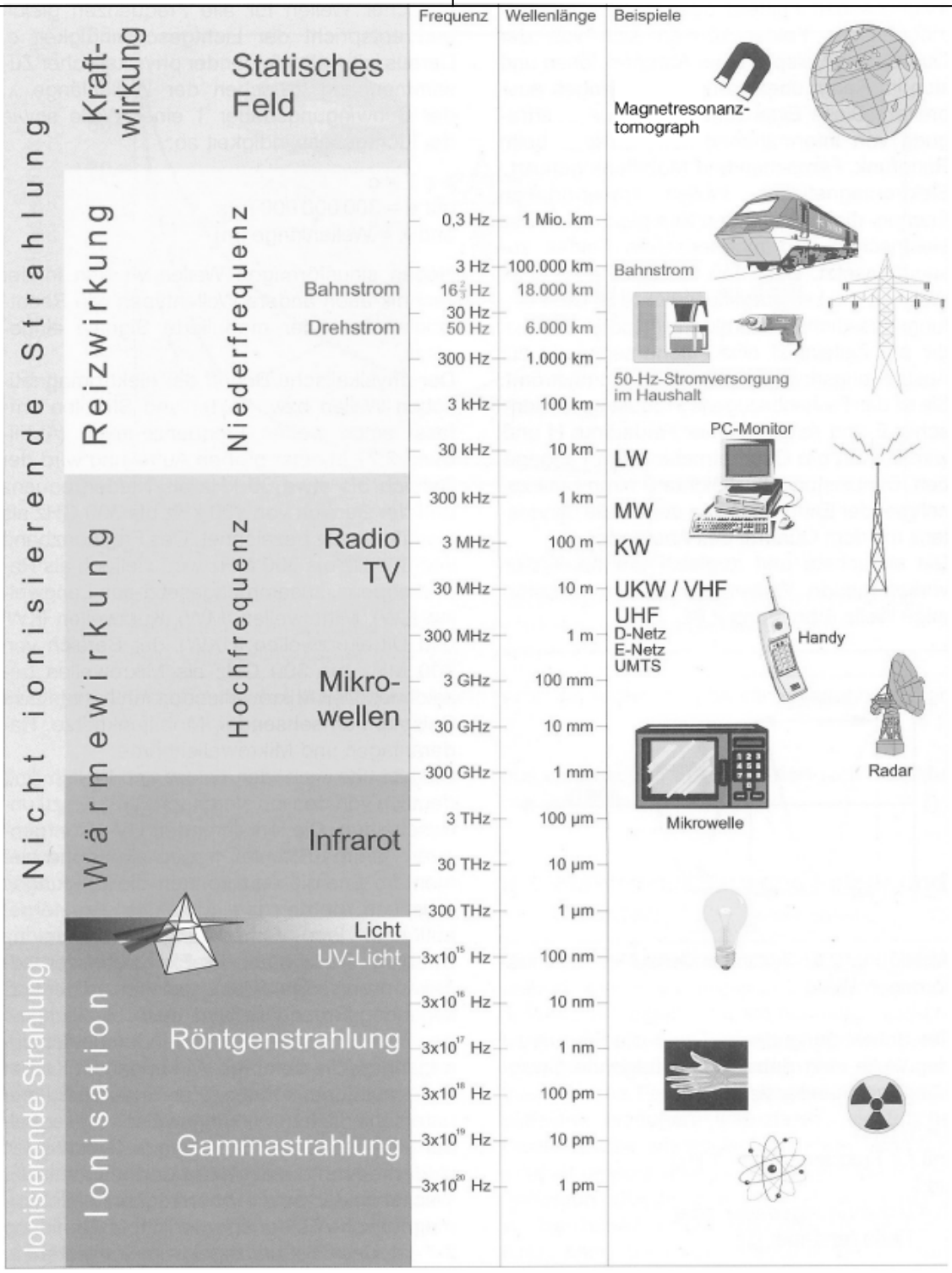

Abb. $7 \Delta$ Frequenzspektrum elektromagnetischer Felder [26]

Gonarthrose-Score (• Abb. 5) hatte sich signifikant $(p<0,0001) \mathrm{zu}$ den leichteren Gruppen gebessert.

Das Department of Orthopaedic Surgery des Changi General Hospital in Singapur untersuchte in einer randomisierten doppelblinden plazebokontrollierten Studie den Effekt der PST auf die Schmerzstärke bei leichten bis mittelschweren Gonarthrosen (Stadium I-III nach Brandt). Insgesamt 41 Patienten wurden in 2 Gruppen randomisiert (20 Patienten in die Verumgruppe mit 9-mal $1 \mathrm{~h}$ PST "lege artis“, 21 in die Plazebogruppe). Die Schmerzstärke wurde mit einer visuellen Analogskala (VAS) von o-10 gemessen. Die Ergebnisse der insgesamt 4 Untersuchungszeitpunkte (vor Therapie/1, 3 und 6 Monate nach
Therapieabschluss, - Abb. 6) wurden einer statistischen Analyse mit dem gepaarten t-Test unterzogen. Die Reduktion der Schmerzstärke in der aktiv behandelten Gruppe war zu allen Untersuchungszeitpunkten mit $p<0,05$ statistisch signifikant. Hingegen zeigte die Plazebogruppe mit $0,61<p<0,72$ keine statistische Signifikanz.

\section{Frequenzspektrum der elektromagnetischen Felder und Signale}

Verschiedene elektromagnetische Felder spielen in unserem Leben eine wichtige Rolle. Sie kommen einerseits natürlich vor, werden andererseits aber auch technisch von uns erzeugt. So findet unsere gesam- te Informationsübertragung mittels elektromagnetischer Signale statt (z. B. Radio, Fernsehen oder Mobilfunk) (• Abb. 7). In der Medizin werden seit Jahren spezielle elektromagnetische Felder diagnostisch (z. B. MRT, Röntgen) oder therapeutisch (z. B. Röntgentiefenbestrahlung oder Radionuklidtherapie) eingesetzt. Seit nunmehr 100 Jahren wird versucht, zunächst mittels Magnetfeldtherapie und später mit gepulsten Magnetfeldern, Einfluss auf Regelungs- und Steuerungsprozesse zu nehmen, z. B. auf Stoffwechsel- oder Durchblutungsstörungen. Gute Erfolge konnten z. B. bei Pseudarthrosen erzielt werden [6].

Die Pulsierende Signal Therapie nutzt einen definierten Ausschnitt des elektromagnetischen Frequenzspektrums, innerhalb dessen Reizwirkungen im menschlichen Körper gesetzt werden können. Das Impulsmuster variiert zwischen 1 und $30 \mathrm{~Hz}$, wobei die Mehrzahl der Signale zwischen 10 und $15 \mathrm{~Hz}$ liegt. Auch die Intensität dieser pulsierenden Signale variiert während der jeweils einstündigen Behandlung fortlaufend, das Maximum der Signalübertragung liegt im Intensitätsbereich von $1,0-1,5 \mathrm{mT}$.

\section{Unterschied zur herkömmlichen Magnetfeldtherapie}

Die Pulsierende Signal Therapie unterscheidet sich von anderen induktiven PEMF-Verfahren (Verfahren mit gepulsten elektromagnetischen Feldern) in den energetischen Parametern durch eine variable Frequenz und Intensität sowie Impulsbreite. Im Gegensatz zur herkömmlichen Magnetfeldtherapie, die wechselstromgenerierte, sinusförmige Impulse anwendet, beruht die PST auf gleichstromgerichteten Rechteckimpulsen, wie sie auch physiologischerweise vorkommen. Eine Untersuchung von 20 unterschiedlichen Geräten zur Erzeugung von therapeutischen Magnetfeldern durch Dipl.-Ing. Dr. Josef Kollmitzer an der Klinik für Physikalische Medizin und Rehabilitation des AKH Wien zeigte, dass Intensität und Bandbreite der untersuchten Geräte deutliche Unterschiede zu den Energieparametern der PST aufweisen [23]. Bei der Behandlung wird durch das elektromagnetische Feld ein spezielles Signalmuster mit auf Bindegewebe abgezielten Energieparametern 
übertragen. Das Patent „Elektromagnetische Stimulation von Knorpelgewebe“ mit Original PST-Energieparametern ist am 23.04.2003 in Europa erteilt worden.

\section{Postulat zum Wirkungsmechanis- mus der PST im Knorpel}

Neben Regulations- und Transkriptionsfaktoren spielen für die Regelung und Steuerung der permanent ablaufenden Umbauund Regenerationsprozesse in den Strukturen des Bewegungsapparats wahrscheinlich feine elektromagnetische Vorgänge eine entscheidende Rolle. Dies sind beispielsweise so genannte „Strömende Potenziale“ im Gelenkknorpel oder „Piezoelektrische Potenziale“ im Knochengewebe wie in der Einleitung beschrieben ( $\bullet$ Abb. 8, 9).

Aufgrund der verminderten Gelenkbelastung (Schonung) und der gestörten Gelenkkinematik (Knorpelverlust, Fehlbelastung, Ausweichbewegungen) werden diese wichtigen Stimulationsvorgänge bei einem geschädigten oder arthrotischen Gelenk nicht mehr in ihrer Gesamtheit hergestellt. Der „Circulus vitiosus“ der zunehmenden Gelenkschädigung und progredienten Knorpelzerstörung kann somit seinen Lauf nehmen.

Das theoretische Modell zum Wirkungsmechanismus lässt sich wie folgt zusammenfassen: Stimulation der verminderten Regenerationsvorgänge im Gelenkknorpel durch Auslösung einer Vielzahl unterschiedlicher „Strömender Potenziale“ im Gelenk während der Therapiestunde (• Abb. 10). Diesen elektrischen Vorgängen im Gelenk kommt in der Steuerung von Regenerationsvorgängen vermutlich eine entscheidende Bedeutung zu und sie sind durchaus den Vorgängen bei physiologischen Gelenkbelastungen vergleichbar.

Eine Messung der auftretenden minimalen elektrischen „Strömenden Potenziale“ im Gelenkbereich während der Therapiestunde ist auch im Tier- oder Zellversuch nicht möglich, da die Messergebnisse von den verabreichten elektromagnetischen Impulsen mehrfach überlagert werden.

\section{In-vitro-Studie zur Wirkung der Pulsierenden Signal Therapie}

In einer im „Labor for Tissue Engineering“ der „Charité“ 2001 durchgeführten Unter-

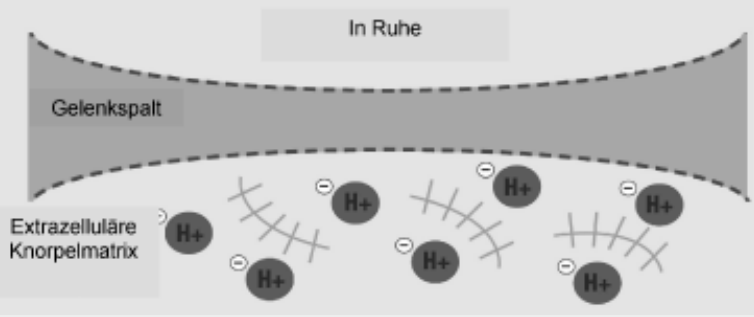

Abb. $8 \Delta$ Gelenk in Ruhe, d. h. Ladungsausgleich zwischen Wasserstoffprotonen und negativen Ladungsträgern der extrazellulären Knorpelmatrix, kein Spannungspotenzial

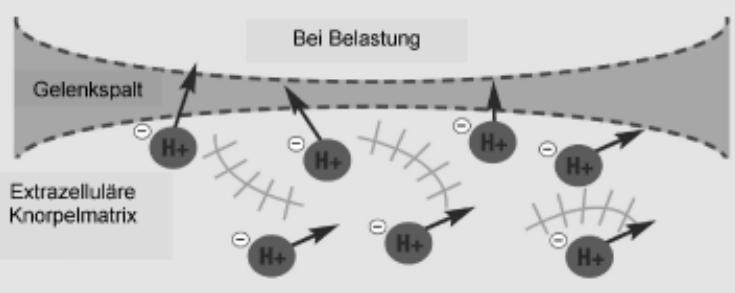

Abb. $9 \Delta$ Gelenk bei Bewegung und Belastung: Vielzahl strömender Potenziale durch Auspressen des Knorpels während der Druckübertragung mit Verschiebung der Wasserstoffprotonen (Durchwalkung des Gelenks)

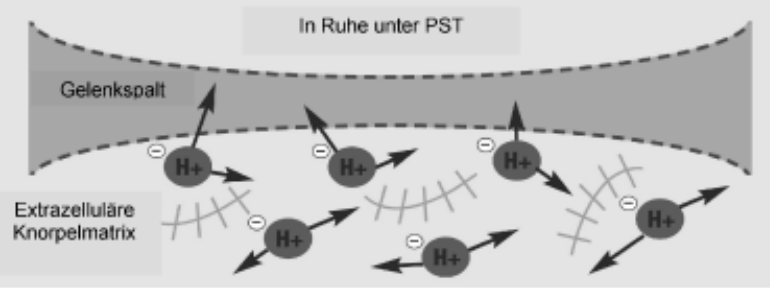

Abb. $10 \triangle$ Gelenk in Ruhe unter PST, wechselnde Rechteckimpulse erzeugen sehr viele, unterschiedliche strömende Potenziale durch die wechselnden Pulsierenden Signale mit Verschiebung der Wasserstoffprotonen in der EZM, Stimulationsreiz für die Zellen des Bindegewebes - in erster Linie Chondrozyten

suchung wurde anhand einer dreidimensionalen Chondrozytenkultur ein Effekt der PST auf die Matrixsynthese von arthrotischen Gelenk- und Meniskuschondrozyten demonstriert [24]. Neben einer Steigerung des Hydroxyprolingehalts wurde eine Größenzunahme der mit PST stimulierten Pellets im Vergleich zu Kontrollpräparaten gefunden.

\section{Fazit}

Die Behandlung mit PST kann eine Erweiterung des konservativen Behandlungsspektrums bei leichten bis mittelschweren Arthrosen darstellen. GCP-konforme (GCP: "good clinical practice") Studien zur Wirk- samkeit, insbesondere auch bei weiteren Indikationen, sind erforderlich. Auch sollte angestrebt werden, im zellbiologischen Verständnis des Wirkungsmechanismus weitere Erkenntnisse zu gewinnen. Hierbei könnten Untersuchungen zur Abklärung einer evtl. gesteigerten Expression knorpelspezifischer Gene unter Pulsierender Signal Therapie neue Ansätze im Verständnis des Wirkmechanismus erbringen.

\section{Korrespondierender Autor}

\section{H. Martin}

Institut für innovative Medizin, Implerstraße 7, 81371 München E-Mail: hm@infinomed.de 
Interessenkonflikt: Der korrespondierende Autor weist auf eine Verbindung mit folgender Firma/Firmen hin: Ärztlicher Leiter Infinomed/ PST - Behandlungszentrum.

\section{Literatur}

1. Aaron RK, Plass AHK (1987) Stimulation of proteoglycan synthesis in articular chondrocyte cultures by a pulsed electromagnetic field. Trans Orthop Res Soc 12: 273

2. Aaron RK, Ciombor DM, Jolly G (1987) Modulation of chondrogenesis and chondrocyte differentiation by pulsed magnetic fields. Trans Orthop Res Soc 12: 272

3. Adey WR (1993) Whispering between cells: electromagnetic fields and regulatory mechanisms in tissue. Frontier Perspect 3: 21-25

4. Armstrong CG, Mow VC (1982) Variations in the intrinsic mechanical properties of human articular cartilage with age degeneration and water content. J Bone Joint Surg Am 64A: 88-94

5. Bassett CAL, Pawluk RJ (1972) Electrical behaviour of cartilage during loading. Science 178: 982-983

6. Brighton CT, Black J, Friedenberg ZB et al. (1981) A multicenter study of the treatment of non-union with constant direct current. J Bone Joint Surg Am 63-A: 2-13

7. Brighton CT, Unger AS, Stambough JL (1984) In vitro growth of bovine articular cartilage chondrocytes in various capacitively coupled electrical fields. J Orthop Res 2: 15-22

8. Cotta H, Wentzensen J, Kramer KL et al. (1995) Standardverfahren in der operativen Orthopädie und Traumatologie und Unfallchirurgie zusammen. Thieme, Stuttgart New York

9. DeWitt MT, Handley CJ, Oakes BW et al. (1984) In vitro response of chondrocytes to mechanical loading, the effect of short-term mechanical tension. Conn Tissue Res 12: 97-108

10. Edwards J (1967) Physical characteristics of articular cartilage. Proc Inst Mech Eng 181-183: 16-24

11. Elliot JP, Smith RL, Block CA (1988) Time-varying magnetic fields: effects of orientation on chondrocyte proliferation. J Orthop Res 6: 259-264

12. Faensen M, Breul R (2001) Prospektive multizentrische Studie zur Behandlung von Gonarthrosen (Kellgren II und III) mit der Pulsierenden Signal Therapie (PST). Orthop Prax 37: 701-709

13. Fioravanti A, Nerucci F, Collodel G et al. (2002) Biochemical and morphological study of human articular chondrocytes in the presence of Pulsed Signal Therapy. Ann Rheum Dis 61: 1032-1033

14. Frank EH, Grodzinsky AJ (1987) Cartilage electromechanics. A continuum model of cartilage electrokinetics and correlation with experiments. J Biomech 20: 629-639

15. Fukuda E, Yasuda I (1957) Piezoelectric effect in bone. J Physiol Soc Jpn 12: 1158

16. Gierse H, Breul R, Faensen M et al. (2000) Pulsed Signal Therapy (PST) stimulates mitosis of human chondrocytes in culture. Proceedings: Tenth International Conference on Biomedical Engineering. Singapore Humanitas Press, Singapore, pp S473474

17. Gossling HR, Bernstein RA, Abbott J (1992) Treatment of ununited tibial fracture: a comparison of surgery and PEMF. Orthopedics 15: 711-719

18. Grande DA, Magee FP, Weinstein AM et al. (1991) The effect of low-energy combined AC and DC magnetic fields on articular cartilage metabolism. Ann NY Acad Sci 635: 404-407
19. Gu WY, Lai WM, Mow VC (1993) Transport of fluids and ions through a porous-permeable charged hydrated tissue, and streaming potential data on normal bovine articular cartilage. J Biomech 26: 709723

20. Gu WY, Lai WM, Mow VC (1997) A triphasic analysis of negative osmotic flows through charged hydrated soft tissues. J Biomech 30: 71-78

21. Gu WY, Lai WM, Mow VC (1998) A mixture theory for charged-hydrated soft tissues containing multielectrolytes: passive transport and swelling behaviors. J Biomech Eng 120: 169-180

22. Horst S (2002) Die Wirkung von PST auf Gelenke und Chondrozyten - klinische und experimentelle Ergebnisse. Med. Dissertation, Universität zu Köln

23. Kollmitzer J (2001) Geräte für Magnetfeldtherapie. Fortbildung, Klinik für Physikalische Medizin und Rehabilitation, AKH Wien, 26.09.2001, http://www. meduniwien.ac.at/phys.med.rehab/jahresbericht. doc

24. Krüger I, Faensen M (2001) The PST effect on 3-dimensional chondrocyte culture: an in vitro study. 1st Biennial Meeting of the Tissue Engineering Society ETES. Symposium of the International Cartilage Repair Society (ICRS), Freiburg, Germany, November 7-10, 2001

25. Lai WM, Hou JS, Mow VC (1991) A triphasic theory for the swelling and deformational behaviors of articular cartilage. J Biomech Eng 113: 245-258

26. Landesanstalt für Umweltschutz Baden-Wuerttemberg (2002) Elektromagnetische Felder im Alltag. Landesanstalt für Umweltschutz Baden-Württemberg, Karlsruhe

27. Linn FC, Sokoloff L (1986) Movement and composition of interstitial fluid of cartilage. Arthritis Rheum 8: 481-494

28. Lipshitz H, Etheredge R, Glimcher MJ (1976) Changes in the hexosamine content and swelling ratio of articular cartilage as functions of depth from the surface. J Bone Joint Surg Am 58A: 1149-1153

29. Liu H, Abbott J, Bee JA (1996) Pulsed electromagnetic fields influence hyaline cartilage extracellular matrix composition without affecting molecular structure. Osteoarthritis Cartilage 4: 63-76

30. Mankin HJ, Thrasher AZ (1975) Water content and binding in normal and osteoarthritic human cartilage. J Bone Joint Surg Am 64A: 76-79

31. Markoll R (2002) Pulsed Signal Therapy: a practical guide for clinicians. In: Weiner RS (ed) Pain management. CRS Press, Boca Raton, Fl, pp 715-728

32. Markoll R, Da Silva Ferreira D (2004) Pulsed Signal Therapy for the treatment of musculoskeletal conditions: a millenium paradigm. APLAR J Rheumatol 7: 287-300

33. Maroudas A (1979) Physicochemical properties of articular cartilage. In: Freeman MAR (ed) Adult articular cartilage. Pitman Medical, Kent, UK, pp 215290

34. Maroudas A, Muir H, Wingham J (1969) The correlation of fixed negative charge with glycosaminoglycan content of human articular cartilage. Biochem Biophys Acta 177: 492-500

35. Mow VC, Kuei SC, Lai WM et al. (1980) Biphasic creep and stress relaxation of articular cartilage in compression: theory and experiments. J Biomech Eng 102: 73-84

36. Perrot S, Marty M, Kahan A et al. (2002) Wirkung der PST Pulsierende Signal Therapie bei schmerzhafter Kniegelenkarthrose. Arthritis Rheum 22 : 101-104

37. Trock DH, Bollet AJ, Markoll R (1994) The effect of pulsed electromagnetic fields in the treatment of osteoarthritis of the knee and cervical spine. J Rheumatol 21: 1903-1911 